\title{
Ultrastrongly dissipative quantum Rabi model
}

\author{
David Zueco \\ Departamento de Física de la Materia Condensada, Instituto de Ciencia de Materiales de Aragon, CSIC-Universidad de Zaragoza, \\ E-50009 Zaragoza, Spain \\ and Fundación ARAID, Paseo María Agustín 36, E-50004 Zaragoza, Spain \\ Juanjo García-Ripoll \\ Instituto de Física Fundamental, IFF-CSIC, Calle Serrano 113b, Madrid E-28006, Spain
}

(Received 24 July 2018; published 3 January 2019)

\begin{abstract}
We discuss both the spectrum and the dynamics of cavity QED in the presence of dissipation beyond the standard perturbative treatment of losses. Using the dynamical polaron ansatz and matrix-product state simulations, we discuss the case where both light-matter $g$ coupling and system-bath interaction are in the ultra-strong-coupling regime. We provide a critical $g$ for the onset of Rabi oscillations. Besides, we demonstrate that the qubit is dressed by the cavity and dissipation. Such a dressing governs the dynamics and, thus, it can be measured. Finally, we sketch an implementation for our theoretical ideas within circuit QED technology.
\end{abstract}

DOI: 10.1103/PhysRevA.99.013807

\section{INTRODUCTION}

More than 80 years ago, Rabi studied the interaction of a two-level system (TLS) with a classical electromagnetic field [1]. Jaynes and Cummings (JC) quantized this theory [2], focusing on the case of a single mode. This model is the quantum Rabi model $(\hbar=1)$,

$$
H_{\mathrm{qR}}=\frac{\Delta}{2} \sigma_{z}+\Omega a^{\dagger} a+g \sigma_{x}\left(a^{\dagger}+a\right) .
$$

In this Hamiltonian, $\Delta$ and $\Omega$ are the bare TLS and cavity frequencies, whereas $g$ denotes the light-matter interaction strength, see Fig. 1(b). Considering the dissipation of the two-level system $\gamma$ and of the cavity $\kappa$, we obtain several light-matter regimes [cf. Fig. 1(a)]. When coupling outweighs dissipation $g \gg\{\gamma, \kappa\}$, the qubit and the cavity field exchange excitations in a coherent way. Here [darker zone in Fig. 1(a)] we distinguish two regimes. If $g / \Delta \lesssim 0.1$, we are in the $S C$ regime, and light-matter interaction can be simplified into $g\left(\sigma^{+} a+\right.$ H.c. $)$ using the RWA. However, if $g / \Delta \gtrsim 0.1$, the RWA fails, and the full interaction, i.e., the counterrotating terms $-g\left(\sigma^{+} a^{\dagger}+\right.$ H.c. $)$-are needed. This is the USC [3,4]. By analogy, when dissipation dominates, we distinguish between the WC and the WUSC, when dissipation dominates, we distinguish between the $W C$ and the WUSC, depending on whether we can apply or not the RWA in the light-matter coupling [light area in Fig. 1(a)].

The goal of this paper is to derive a mathematical treatment of the cavity-QED model that provides quantitatively or qualitatively accurate solutions in all coupling and dissipation regimes-WC, SC, WUSC, and USC - . There are many ways to solve the cavity-QED model that apply to subsets of these regimes. In the absence of dissipation, Eq. (1) admits an analytical solution [5] and can be solved in the computer for any $g$ value. If losses are taken into account, they are typically discussed using Markovian master equations [6,7]. They are perturbative in the system-bath interaction $[8,9]$.
Going beyond this perturbative treatment is tricky [10]. Renormalization, path-integral expansions, or numerical techniques are required $[11,12]$. In contrast to this zoo of solutions, we will offer a different method of broad utility with the only restriction that qubit dissipation remains below the quantum phase transition into the strongly correlated regime. The resulting method will be useful in studying all the quantum technologies that are developed around the JC mode- singlephoton emitters, quantum computers, spin squeezing [13]-, as well as experiments that exploit the huge dipole moments of superconducting qubits in the ultra-strong-coupling regime [14-16].

Our method builds on the polaron Hamiltonian $[17,18]$ to develop an effective model that can be analytically or numerically solved. Similar to the Ohmic spin-boson theory [19-21], we predict non-Markovian renormalization of the qubit splitting $\Delta$ due to the coupling with the bath either directly $\gamma$ or via the cavity. We can also solve the qubitcavity dynamics from overdamped decay in the limit of WC or WUSC dissipation to coherent scenarios that extend well inside the USC both in losses and in light-matter coupling, see Fig. 1.

The outline of this paper is as follows. In Sec. II, we summarize the model, the polaron transformation, and the different ways of solving the spectrum, and the dynamics. In Sec. III, we announce our results. We discuss the ground-state properties of the model, the onset of Rabi oscillations, and the noise spectrum. Finally, we give some conclusions and a possible implementation in Sec. V. Several technical details are discussed in the appendices.

\section{THEORETICAL METHODS}

\section{A. Combined dissipation channels}

We consider a qubit and a cavity interacting with each other and coupled to independent baths. The model in the 
(a)

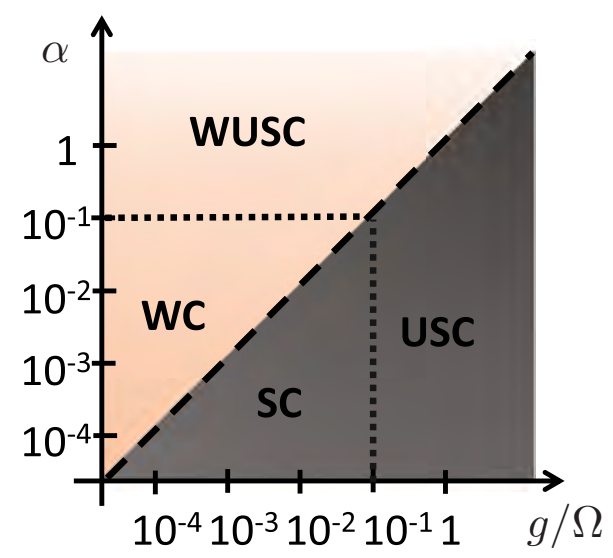

(b)

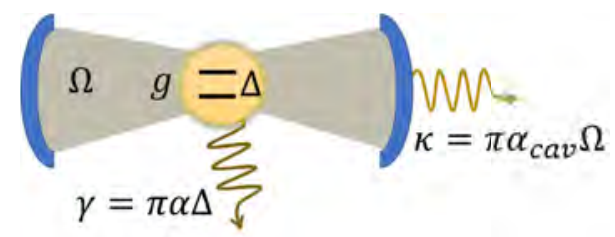

FIG. 1. Cavity QED phase diagram and setup sketch. (a) Dark region marks where Rabi oscillations occur. We distinguish between strong-coupling (SC) and ultra-strong-coupling (USC) regimes depending if the rotating-wave approximation (RWA) between the cavity field and the atom can be performed or not. In the light region, losses are big enough, and the TLS decays in an overdamped way. Consistently, we must distinguish between weak coupling (WC) and weak ultra-strong-coupling (WUSC) regimes. In the figure $\alpha$ characterizes the TLS losses [cf. Eq. (3a)]. In (b) we draw a cavity QED sketch with the main parameters indicated.

system-bath formalism [22,23] is [cf. Eq, (1)] as follows:

$$
\begin{aligned}
H= & H_{\mathrm{qR}}+\sum_{i=1,2} \sum_{k}^{N} \omega_{k, i} b_{k, i}^{\dagger} b_{k, i}+\sigma_{x} \sum_{k}^{N} c_{k, 1} X_{k, 1}+\left(a+a^{\dagger}\right) \\
& \times \sum_{k}^{N} c_{k, 2} X_{k, 2}+(a+a)^{2} \sum_{k} \frac{\left|c_{k}\right|^{2}}{2 \omega_{k}}
\end{aligned}
$$

The qubit and cavity baths have independent modes $\omega_{k, i}(i=$ 1,2) with bosonic quadratures $X_{k, i} \equiv b_{k, i}^{\dagger}+b_{k, i}$. Both noise channels can be described using spectral density functions $J_{i}(\omega)=2 \pi \sum_{k} c_{k, i}^{2} \delta\left(\omega-\omega_{k, i}\right)$. In this paper, we are considering an Ohmic noise spectrum for both the cavity and the spin, i.e., $J_{i}(\omega) \sim \omega$. In the Markovian limit, the dissipation strength is determined by the spontaneous emission rates of the qubit $(\gamma)$ and the cavity $\kappa$ because [cf. Fig. 1(b)]

$$
\begin{aligned}
& \gamma=J_{1}(\Delta)=\pi \alpha \Delta, \\
& \kappa=J_{2}(\Omega)=\pi \alpha_{\mathrm{cav}} \Omega .
\end{aligned}
$$

with $\alpha\left(\alpha_{\text {cav }}\right)$ as dimensionless parameters characterizing the dissipation strength for the TLS (cavity).

The last term in Eq. (2) deserves some discussion. This regularization of the bosonic modes arises from a cavity-bath coupling of the form $\sim\left(a+a^{\dagger}-\Phi\right)^{2}$, where $a+a^{\dagger}$ is the cavity quadrature and $\Phi$ is the electromagnetic field injected by the bath. This type of coupling-which is very natural in superconducting circuits-ensures that the total energy is bounded from below and leads to the quadratic correction of the bosonic modes. Importantly, the quadratic correction ensures that the resonance of the cavity stays at $\Omega$, irrespective of the dissipation strength $\alpha_{\text {cav }}$. Note also that we do not find a similar term in the qubit-bath coupling because of saturation: $\left(\sigma^{x}\right)^{2}=1$. Further parameter renormalization is associated with quantum many-body effects between the bath and the cavity QED system [10,24].

The cavity mode in (1) can be diagonalized together with its environment. In doing so, Hamiltonian (2) is rewritten as a spin-boson model [19] for a two-level system coupled to two baths, one of which contains the cavity mode,

$$
H=\frac{\Delta}{2} \sigma_{z}+\sigma_{x} \sum_{k^{\prime}}^{2 N+1} c_{k^{\prime}}\left(b_{k^{\prime}}^{\dagger}+b_{k^{\prime}}\right)+\sum_{k^{\prime}}^{2 N+1} \omega_{k^{\prime}} b_{k^{\prime}}^{\dagger} b_{k^{\prime}} .
$$

By joining the cavity modes and the qubit bath, we arrive at the total spectral density $J(\omega)=2 \pi \sum_{k^{\prime}} c_{k^{\prime}}^{2} \delta\left(\omega-\omega_{k^{\prime}}\right)$,

$$
J(\omega)=\pi \alpha \omega+\frac{4 g^{2} \pi \alpha_{\mathrm{cav}} \Omega^{2} \omega}{\left(\Omega^{2}-\omega^{2}\right)^{2}+\left(\pi \alpha_{\mathrm{cav}} \Omega \omega\right)^{2}} .
$$

The second term characterizes the bath containing the cavity mode and is peaked around the cavity frequency $\Omega$ [25]. The first term accounts for the intrinsic qubit Ohmic environment. We will correct this spectral function by introducing a hard cutoff $\omega_{c}$ [20].

Finally, note that models (2) and (4) are completely equivalent. When writing the model as (4), Rabi oscillations can be understood as non-Markovian decaying oscillations coming from the peaked spectral density. Details on the cavity-bath diagonalization and the effective spectral density are given in Appendix B.

\section{B. Effective RWA models}

It has been recently shown that the low-energy spectrum of a spin-boson model (5) can be very well approximated by an effective excitation number conserving Hamiltonian derived from a polaron transformation $[17,18]$. The basic idea is to construct a unitary transformation that disentangles the TLS from the bath,

$$
U_{p}=\exp \left[\sigma_{x} \sum\left(f_{k} b_{k}^{\dagger}-f_{k}^{*} b_{k}\right)\right]
$$

and choosing the displacements $f_{k}$ with the Silbey-Harris prescription that the ground state of $H_{p}=U_{p}^{\dagger} H U_{p}$ be as close as possible to $|0\rangle \otimes|\mathbf{0}\rangle$ the ground state of the uncoupled TLS $|0\rangle$ and of the bath $|\mathbf{0}\rangle$. Minimization yields the self-consistent relation,

$$
f_{k}=\frac{-c_{k} / 2}{\Delta_{r}+\omega_{k}} \quad \text { with } \Delta_{r}=\Delta \exp \left(-2 \sum_{k} f_{k}^{2}\right),
$$


and the effective Hamiltonian $H_{p}$ is well approximated within the single-excitation sector by [26]

$$
\begin{aligned}
H_{p 1} \cong & \frac{\Delta_{r}}{2} \sigma_{z}+\sum_{k}^{N} \omega_{k} b_{k}^{\dagger} b_{k}-2 \Delta_{r}\left(\sigma^{+} \sum_{k}^{N} f_{k} b_{k}+\text { H.c. }\right) \\
& -2 \Delta_{r} \sigma_{z} \sum_{k, p}^{N} f_{k} f_{p} b_{k}^{\dagger} b_{p} .
\end{aligned}
$$

This model has two important features. First, our TLS appears with the renormalized frequency $\Delta_{r}$, that determines the dynamics of the qubit in the bath [cf. Sec. III B]. Second, the model develops a conserved quantity $\left[H_{p}, \sigma_{z}+\sum_{k} b_{k}^{\dagger} b_{k}\right]=0$ and becomes tractable with the same techniques as the RWA models.

\section{Estimating $\Delta_{r}$}

The qubit renormalized frequency $\Delta_{r}$ [cf. Eqs. (7) and (8)] admits analytical solutions in the continuum limit where we can write

$$
\Delta_{r}=\Delta \exp \left[-1 / 2 \int_{0}^{\omega_{c}} J(\omega) /\left(\omega+\Delta_{r}\right)^{2}\right],
$$

using the UV cutoff $\omega_{c}$. The resulting expression is not tractable and requires the numerical solution of a transcendental equation for $\Delta_{r}$ in (9). One extreme limit of this equation appears when the qubit decouples from the cavity $(g=0)$. In that case Eq. (9) can be solved, resulting in the Ohmic spin-boson model. Then, $\Delta_{r}=\Delta\left(\Delta / \omega_{c}\right)^{\alpha /(1-\alpha)}$, and the localization-delocalization transition at $\alpha=1$ [19]. When we depart from this limit $g \neq 0$, the second summand in (5) decreases the onset of the localization transition to lower values of $\alpha$.

The predictions of the polaron ansatz can be compared with the adiabatic renormalization group (ARG) [19] in the continuum limit. Applied to the spin-boson model, the ARG predicts a qubit frequency at

$$
\Delta_{r}^{\mathrm{ad}}=\Delta \exp \left[-1 / 2 \int_{\Delta_{r}}^{\omega_{c}} J(\omega) / \omega^{2}\right]
$$

Comparing (9) and (10) we see a difference in the lower limit of the integral. The reason is that the RG flow stops at $\omega_{c} \sim \Delta_{r}$. If $g=0$, both methods yield the same result except corrections on the order of $O\left(\Delta_{r} / \omega_{c}\right)$. Therefore, they predict the same $\Delta_{r}$, however, if $g \neq 0$, the ARG is different from a polaron (and becomes less accurate). Our interpretation is that the cavity $\Omega$ behaves as an effective cutoff and for $\Delta \sim \Omega$ the ARG should fail. More generally, we also expect the ARG to fail whenever cavity losses and/or light-matter coupling dominate over the TLS intrinsic dissipation.

\section{Modified Wigner-Weisskopf}

We have just discussed that (8) conserves the number of excitations. We can solve the dynamics within a single-excitation subspace à la Wigner-Weisskopf. There, the dynamics is fully determined by the wave function,

$$
|\Psi(t)\rangle=U_{p}\left[f_{k}\right]\left(\psi \sigma^{+}+\sum_{k} \psi_{k} b_{k}^{\dagger}\right)|0, \mathbf{0}\rangle .
$$

Using (8) and (11), the coefficients $\left\{\psi, \psi_{k}\right\}$ satisfy the set of coupled linear equations,

$$
\begin{aligned}
\dot{\psi} & =-i 2 \Delta_{r} \sum \psi_{k} f_{k}, \\
\dot{\psi}_{k} & =-i\left(\omega_{k}-\Delta_{r}\right) \psi_{k}+i 2 \Delta_{r} f_{k}\left(\psi-\sum_{k^{\prime}} f_{k^{\prime}} \psi_{k^{\prime}}\right) .
\end{aligned}
$$

From these coefficients we may derive, for instance, the excitation probability of the two-level system,

$$
P_{e}=\frac{\left\langle\psi(t)\left|\sigma_{z}\right| \psi(t)\right\rangle+1}{2} .
$$

In the regime $g / \Delta, \alpha, \alpha_{\text {cav }} \ll 1$, we can solve for the qubit amplitude $\psi$ applying the Markov approximation on the qubit losses and replacing the second summand in (5) with a Lorentzian centered on the cavity resonance $\Omega$. Then an analytical solution is possible as it is fully developed in our Appendix C. In the ultrastrong coupling, both for losses and light-matter coupling, analytical advances are possible in the calculation of the qubit noise spectrum $S(\omega)$ as we explain in Sec. III C. However, for the time evolution, in general, an analytical solution is no longer possible. Then, we approximate the environment using a finite number of modes $N$ as explained in Appendix A. In doing so, we can solve the set of $O(N)$ ordinary differentials (12a) and (12b) numerically, e.g., using Lanczos, Runge-Kutta, or any other available method.

\section{E. Matrix-product states}

In order to confirm the predictions of $H_{p}$, we also run numerical simulations on the unapproximated model $H_{p}=$ $U_{p}^{\dagger} H U_{p}$ using a matrix-product state (MPS) ansatz. Whereas working with $H_{p}$ significantly decreases the amount of entanglement in the MPS simulation, we introduce another optimization and express $H_{p}$ as a tight-binding model [27]. The simulated model reads

$$
\begin{aligned}
H_{p}= & \frac{\Delta_{r}}{2} \sigma_{z} e^{-\theta c_{0}^{\dagger}} e^{\theta c_{0}}+2 \Delta_{r} \theta \sigma_{x}\left(c_{0}+c_{0}^{\dagger}\right) \\
& +\sum_{i}\left(\beta_{i} c_{i+1}^{\dagger} c_{i}+\beta_{i} c_{i}^{\dagger} c_{i+1}+\alpha_{i} c_{i}^{\dagger} c_{i}\right),
\end{aligned}
$$

where $\theta^{2}=\sum_{k} f_{k}^{2}$. The new collective modes $c_{i}$ are constructed from the original ones $b_{i}$ using a Lanczos recursion [27] that also produces the real numbers $\alpha_{i}$ and $\beta_{i}$. This new model has the advantage that it can be simulated using both Arnoldi- and Trotter-type MPS methods [28].

\section{CAVITY-QED BEYOND THE MARKOVIAN REGIME}

\section{A. Ground state}

We will now show how to apply the previous formalism to study the equilibrium properties and dynamics of the cavityQED setup in all regimes. We begin with the nature and properties of the ground state. 

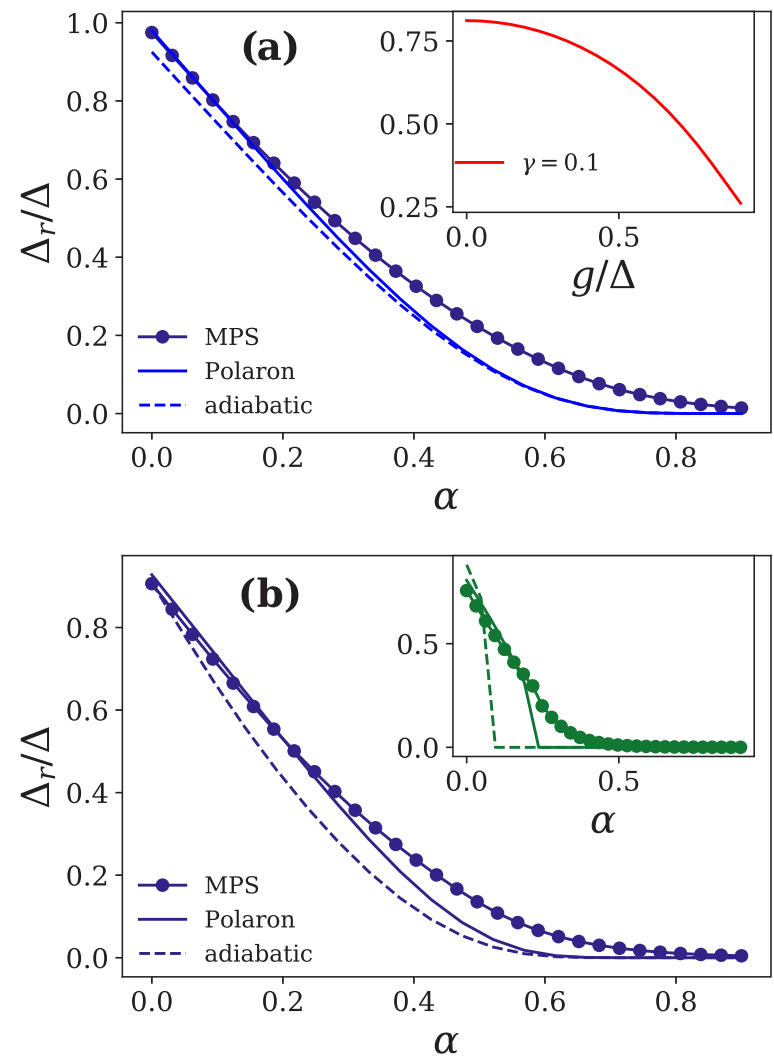

FIG. 2. Frequency renormalization $\left(\Delta_{r}\right)$. In (a) we compare the prediction of our three theories: polaron, adiabatic RG, and the MPS numerical simulation as a function of $\alpha$. We set $\kappa=\pi \alpha_{\text {cav }}=0.01 \pi$ and $g=0.2$. In the inset we show the dependence on $g$ strength for $\alpha=0.1$ and the same $\kappa$. In (b) we do the same but setting $\kappa=\pi 0.8$ and $g=0.4$ (main panel) and $\kappa=\pi 1.5$ and $g=0.6$ (inset). In all the figures the cavity and qubit bare parameters are $\Omega=\Delta=1$.

The construction of the polaron Hamiltonian provides a zeroth-order approximation to the ground-state $U_{p}|0, \mathbf{0}\rangle$, which predicts that the qubit has some probability to be excited. This is consistent with earlier findings in lossless cavities $[8,29]$ and in the spin-boson model without cavities [19], but our treatment allows us to interpolate between both limits. The equilibrium $z$ magnetization is proportional to the qubit renormalized frequency,

$$
\left\langle\sigma_{z}\right\rangle_{\mathrm{eq}}=\left\langle 0, \mathbf{0}\left|U_{p}^{\dagger} \sigma_{z} U_{p}\right| 0, \mathbf{0}\right\rangle=-\frac{\Delta_{r}}{\Delta},
$$

which, as we saw before, can be computed from the displacements $f_{k}$ (7).

Let us now compare the estimates of $\left\langle\sigma_{z}\right\rangle$ and $\Delta_{r}$ from the adiabatic renormalization-group (10), the polaron method Eq. (9), and an exact solution of $H_{p}$ with the MPS discussed in Secs. II C and IIE. Figure 2 summarizes the ground-state properties for different values of the dissipation and coupling strength. Those simulations have been performed with $N=$ 256 modes for the cavity bath and a similar amount for the qubit bath, ensuring numerical convergence to a quasicontinuum limit. In panel 2(a) we plot the qubit renormalization $\Delta_{r}$ as a function of the TLS dissipation [ $\alpha$, cf. Eq. (3a)] for bare parameters $\Omega=\Delta=1$, a USC coupling strength $g=0.2$, and a low cavity spontaneous emission $\kappa=\pi \alpha_{\text {cav }} \Omega=\pi 0.01$. We compare three methods: the polaron, the ARG, and the MPS simulations as explained in Sec. II. The dependence of $\Delta_{r}$ on $\alpha$ resembles the pure Ohmic spin-boson model. As we have anticipated and explained in Sec. II C, the ARG is not accurate for small TLS intrinsic noise strength $\alpha$. The inset of Fig. 2 also shows that the qubit-cavity coupling lowers even further the qubit frequency $\Delta_{r}$ due to the friction induced by the additional bosonic modes from the cavity and its bath.

Figure 2(b) shows the ground state for stronger cavity dissipation, entering the WUSC regime. As seen in the main panel, for relatively high cavity losses $\left(\kappa=\pi \alpha_{\text {cav }} \Omega=\pi 0.8\right)$ the trend of $\Delta_{r}$ is qualitatively similar to the uncoupled case $g=0$. However, if we increase $\kappa$ (and $g$ ) enough, both the polaron and the ARG models predict a sharp transition, leading to localized solutions $\Delta_{r}=0$. Since the MPSs are numerically exact simulations and do not exhibit such a transition, we conclude that this is an artifact of the polaron method that constrains its applicability to large values of dissipation and light-matter coupling $g \geqslant 0.6$ and $\kappa \geqslant 1$. The remaining of this paper will stay well within this regime in which simulations verify well against the MPS.

\section{B. Nonperturbative Rabi oscillations}

In this section we study the dynamics of the cavity-QED setup, solving numerically and analytically the qubit excitation probability $P_{e}$ (13) with the polaron methods detailed in Sec. II D and in Appendix C. The first result is the evidence of coherent light-matter (Rabi) oscillations that: (i) are resonant around the qubit renormalized frequency $\Delta_{r}$ and (ii) dampen exponentially with a modified spontaneous emission rate,

$$
\gamma_{r} \simeq J\left(\Delta_{r}\right)
$$

determined by the joint spectral function (3a) that we introduced in Sec. II. We find that Rabi oscillations start approximately at the boundary,

$$
g \cong\left|\kappa-\gamma_{r}\right| / 4 .
$$

Above this critical value, the TLS and the cavity exchange excitations coherently; below this boundary, the qubit exhibits overdamped exponential decay without oscillations. It is remarkable that this boundary is formally the same as the one in the RWA and Markovian approximation $\left(g / \Omega, \alpha, \alpha_{\text {cav }} \ll 1\right.$ regime) [30] but extends to the USC and WUSC regimes, which do not admit a perturbative treatment.

In Fig. 3 we show the qubit dynamics for varying $g$. In our calculations we start with the qubit in the groundstate $U_{p}|0, \mathbf{0}\rangle$ and perturb it with a $\pi$ pulse, obtaining state $|\Psi(0)\rangle=\sigma_{x} U_{p}|0, \mathbf{0}\rangle=U_{p} \sigma_{x}|0, \mathbf{0}\rangle=U_{p} \sigma^{+}|0, \mathbf{0}\rangle$. Note that this corresponds to $\left\{\psi=1, \psi_{k}=0\right\}$ in Eqs. (12a) and (12b).

In Fig. 3(a) we see the appearance of oscillations for $g \gtrsim$ $\left|\gamma_{r}-\kappa\right| / 4 \cong 0.1$. These plots confirm that the TLS dynamical frequency is given by $\Delta_{r}$ since this resonance changes with $g$, we explore on- and off-resonant oscillations as we increase the coupling strength for fixed $\Omega$. With the parameters used, $\Omega=\Delta_{r}=0.68$, which is reached at $g=0.3$. Figure $3(\mathrm{~b})$ shows the qubit dynamics at precise couplings. This includes the resonant case $g=0.3$, which shows resonantlike Rabi oscillations in the middle plot. For lower coupling $g=0.05$, 

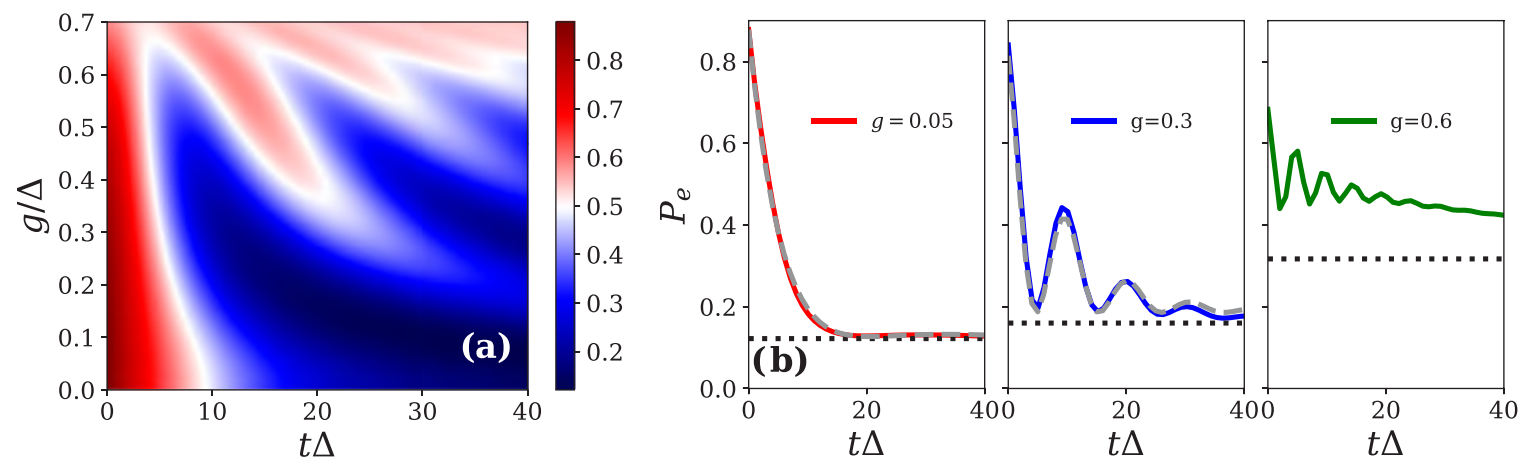

FIG. 3. Rabi oscillations. Panel (a) is a contour for $P_{e}(t)$ as a function of the light-matter coupling. The parameters used are $\kappa=\pi 0.01 \Omega$ and $\Omega=0.68$. This value for the cavity frequency is chosen for being in resonance with the renormalized qubit frequency $\Delta_{r}$ when $g=0.3$ [see the inset of Fig. 2 (a)]. In (b) we plot three cuts for $g=0.05,0.3$, and 0.6. The bare TLS frequency $\Delta=1$. The gray dashed lines correspond to the approximate formula Eq. (18). The horizontal black dotted lines stand for the equilibrium value $P_{e}^{\text {eq }}=\left(1-\Delta_{r} / \Delta\right) / 2$.

losses dominate, and the TLS dynamics is overdampedi.e., exponential decay in the polaron frame-. Since for $\alpha=0.1, \Delta_{r} \neq \Delta$, this is an example of WUSC dynamics. The right-hand plot shows a USC coupling dynamics with $g=0.6$, where $\Delta_{r} \cong 0.4$ and the dynamics are nonresonant Rabi-like oscillations.

We have found an analytical approximation that reproduces and explains the TLS-cavity dynamics and the onset of the Rabi oscillations. We take Eq. (5), remove the term that is $O\left(f^{2}\right)$, and modify the rest, replacing the effective displacements $f_{k}$ with the original couplings $f_{k} \rightarrow c_{k}$. The solution of these simplified equations is formally identical to the one for $g / \Delta, \alpha, \alpha_{\text {cav }} \ll 1$ (Markov and Lorentzian approximations) but with a renormalized frequency $\Delta \rightarrow \Delta_{r}$. We denote it as $\widetilde{P}_{e}(t)$. Besides, we need to impose that the time converges in $t \rightarrow \infty$ to the correct equilibrium solution given by $P_{e}^{\text {eq }}=$ $\left(1-\Delta_{r} / \Delta\right) / 2$ [cf. (13) and (15)]. Note that our numerical simulations verified thermalization, marked as the dotted lines in Fig. 3. To have the correct stationary limit, we use the simplest interpolation for our analytical estimation,

$$
P_{e}^{\mathrm{app}}(t) \cong\left(1-P_{e}^{\mathrm{eq}}\right) \widetilde{P}_{e}(t)+P_{e}^{\mathrm{eq}} .
$$

In Fig. 3(b) we show how such an approximation holds for relatively high $g$ (well inside the ultra-strong-coupling regime), justifying Eq. (17). At strong-coupling $g=0.6$, the simple approximation $P_{e}^{\text {app }}$ stops working (not shown) because we have neglected the $O\left(f^{2}\right)$ terms. In any case, the evolution still reflects detuned Rabi oscillations, converging to the expected limit: $P_{e}(t \rightarrow \infty) \rightarrow \frac{1}{2}\left(\Delta_{r} / \Delta-1\right)$.

We further verified the location of the critical value (17), analyzing numerically the transition from an overdamped dynamics to the appearance of the first oscillations. For that we compute the maximum of the time derivative of $P_{e}$ on the time-interval $[0, T]$ with $T$ sufficiently large. We denote this quantity as $\max d P_{e} / d t$. If the TLS is overdamped, then $d P_{e} / d t<0$ always, and $\max d P_{e} / d t=0$. However, if some oscillations occur, the derivative is sometimes positive. This is represented in Fig. 4 and compared with the bound (17), exhibiting very good agreement. Two comments are in order. First, to generate Fig. 4 we have chosen to be approximately at resonance at the critical value of $g$. Second, since $\Delta_{r}$ can go to zero faster that linearly with $\alpha, \gamma_{r}$ approaches zero by increasing $\alpha$. In the figure, we indeed see that $\alpha_{r}(\alpha=0.2) \cong$ $\alpha_{r}(\alpha=0.3)$ [cf. Fig. 4].

Summing up, our simulations justify the use of quantum optics approximations in this nonperturbative regime. We find that Rabi oscillations extend qualitatively into a regime where light-matter interaction and dissipation are both nonperturbative. In particular, we have studied a regime-denoted the WUSC-in which $g$ is big enough that we cannot make the RWA (the definition of the USC), but at same time, losses are also large and prevent coherent exchange between light and matter degrees of freedom (the definition of weak coupling) [cf. Fig. 1].

\section{Qubit noise spectrum}

The TLS emission spectrum $S(\omega)$ is a very useful experimental tool that provides information about the coupling $g$ and the TLS linewidth. $S(\omega)$ is typically computed using the input-output formalism [31]. In this framework, output and input fields are related to the TLS state via $a_{\text {out }}=a_{\text {out }}-$ $i \sqrt{\Gamma_{\mathrm{TLS}}} X^{-}(t)$, where $\Gamma_{\mathrm{TLS}}$ is the emission rate into the transmission line in which the output signal is collected and $X^{-}$is

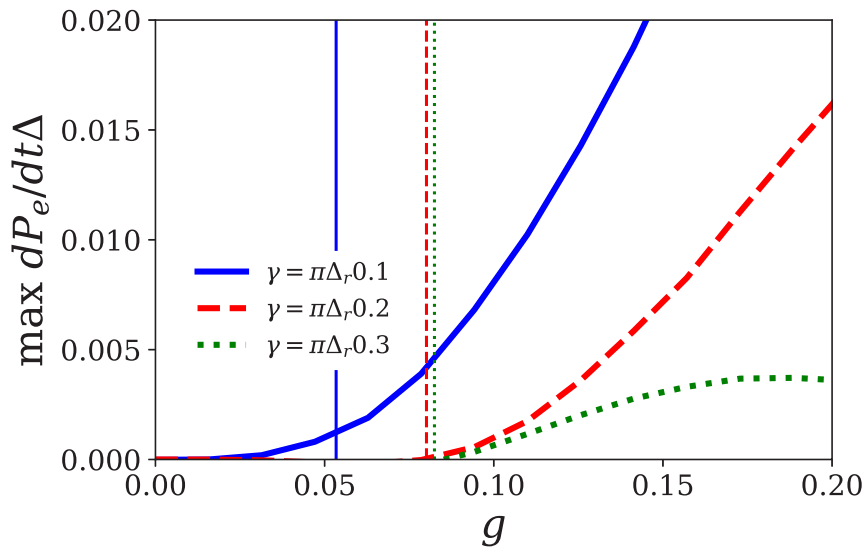

FIG. 4. Weak-coherent coupling. We plot the maximum derivative of the qubit excitation probability max $d P_{e} / d t$ as a function of $g$ for different noise strengths (solid lines). This derivative is negative or zero in the overerdamped regime. The vertical dashed lines mark the prediction for the critical $g$ given by (17). Equal colors mean equal parameters. 


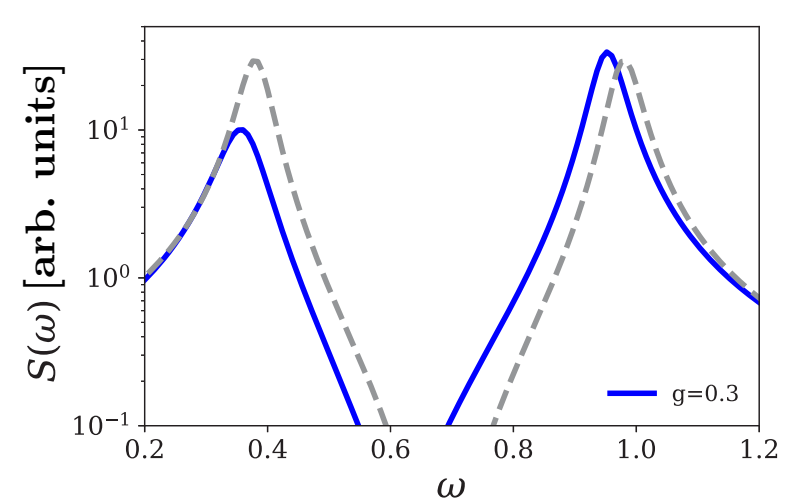

FIG. 5. $S(\omega)$ at resonance $\left(\Delta_{r}=\Omega\right)$. The parameters are those of Fig. $3 g=0.3, \alpha=0.1, \Omega=\Delta_{r}=0.68$, and $\kappa=\pi 0.01 \Omega$. The blue lines stand for $S(\omega)$ calculated with Eqs. (20a) and (20b). The gray dashed lines are calculated with the approximations (21a) and (21b).

the negative frequency component of the qubit-TL coupling operators ( $\sigma_{x}$ in our case) $[32,33]$. The emission spectrum is defined as $S(\omega)=\int_{0}^{\infty} d t \int_{0}^{\infty} d t^{\prime} e^{-i \omega\left(t-t^{\prime}\right)}\left\langle X^{+}(t) X^{-}\left(t^{\prime}\right)\right\rangle$. Since $H_{p}$ is number conserving, $S(\omega)$ can be calculated directly from the Laplace transform $\alpha(s)$ in (12a). After some algebra (fully specified in Appendix D), we end up with

$$
S(\omega) \sim \frac{1}{\left[\omega-\Delta_{r}-R(\omega)\right]^{2}+\Gamma(\omega)},
$$

where $R(\omega)$ and $\Gamma(\omega)$ are the real and imaginary parts of the self-energy of the qubit. The former gives the position of the eigenvalues, and the latter gives the linewidth. Their explicit expressions are

$$
\begin{aligned}
& R(\omega)=\frac{2 \Delta_{r}\left[\left(\mathcal{K}^{\prime}\right)^{2}+\left(\mathcal{K}^{\prime \prime}\right)^{2}\right]-2 \Delta_{r} \mathcal{K}^{\prime \prime}}{\left[\left(2 \Delta_{r}\right)^{2}-\mathcal{K}^{\prime \prime}\right]^{2}+\left(\mathcal{K}^{\prime}\right)^{2}} \\
& \Gamma(\omega)=\frac{\left(2 \Delta_{r}\right)^{2} \mathcal{K}^{\prime}}{\left[\left(2 \Delta_{r}\right)^{2}-\mathcal{K}^{\prime \prime}\right]^{2}+\left(\mathcal{K}^{\prime}\right)^{2}},
\end{aligned}
$$

and $\mathcal{K}^{\prime}\left(\mathcal{K}^{\prime \prime}\right)$ is the real (imaginary) part of

$$
\mathcal{K}=-i\left(2 \Delta_{r}\right)^{2} \int_{0}^{\infty} \frac{J(v)}{\left(v+\Delta_{r}\right)^{2}} \frac{1}{(v-\omega)-i 0^{+}} d v .
$$

We note that the renormalized frequency $\Delta_{r}$ is again explicit. If $\alpha, \alpha_{\text {cav }}, g / \Delta \ll 1$, the linewidth reduces to

$$
\begin{aligned}
& \Gamma(\omega)=\frac{g^{2} \pi \alpha_{\mathrm{cav}} \Omega / 2}{(\omega-\Omega)^{2}+\left(\pi \alpha_{\mathrm{cav}} \Omega / 2\right)^{2}}+\pi \alpha \Delta, \\
& R(\omega)=\frac{g^{2}(\omega-\Omega)}{(\omega-\Omega)^{2}+\left(\pi \alpha_{\mathrm{cav}} \Omega / 2\right)^{2}}
\end{aligned}
$$

recovering the standard results in cavity QED (using, e.g., master equations for dealing with the bath [34]).

The expressions (20a) and (20b) evidence important corrections in the response profile with the most evident fact of an asymmetry between peaks at the dressed resonance $\Omega=\Delta_{r}$ [cf. Fig. 5]. This is a signature of the modified coupling constants $c_{k} \rightarrow f_{k}$ in the nonperturbative polaron Hamiltonian. From Eq. (7) the effective coupling coefficients $f_{k}$ are smaller than $c_{k}$ for $\omega_{k}<\Delta_{r}$ and bigger in the region of the spectrum, leading to the asymmetric profiles. A similar phenomenon has been identified in the USC regime, which accounts for large $g$ but only for weak dissipation [35].

In the previous section we tested a simple approximation to the TLS dynamics (13). This consisted in taking the RWA, weak-, and strong-coupling solutions-i.e., $\alpha, \alpha_{\text {cav }}, g / \Delta \ll$ 1 - but replacing the qubit resonance $\Delta \rightarrow \Delta_{r}$ and correcting for the equilibrium state. If we use this approximation to estimate $S(\omega)$, we find that it more or less accounts for the linewidths. However, our qualitative method fails to reproduce the asymmetry of the peaks [cf. Fig. (5)] and has a minor error in the peak location due to the the Bloch-Siegert and dissipation-induced shifts.

\section{CIRCUIT QED IMPLEMENTATION}

The model that we have discussed in this paper admits a straightforward realization using superconducting circuits. The three elements that we need are as follows: (i) a qubit that is ultrastrongly coupled to the cavity [14,15], (ii) a possibly ultrastrong coupling between the same qubit and some external environment, such as a transmission line [21], and (iii) a strong or ultrastrong coupling between the superconducting cavity and its own bath, a regime already achieved in Ref. [36].

All these three elements admit full and independent tunability, probing arbitrary values of $g, \kappa$, and $\gamma$. First of all, the coupling of the qubit to the photons - $g$ and $\gamma$ in our modelcan be tuned using superconducting quantum interference devices (SQUIDs) that can be embedded in the design of the qubit itself [20,37] as demonstrated in Ref. [21] for a flux qubit in an open transmission line. Moreover, the coupling between the cavity and its own bath $\kappa$ can also be adjusted using in-line dc SQUIDs. This has been demonstrated in the laboratory and used for photon trapping and release [38,39]applications that are much more demanding than the simple stationary tuning of the parameter $\kappa$ in our model.

Assuming superconducting circuit implementations, we can probe the physics of the combined environments in various ways. We have studied the spectral function $J(\omega)$, which can be reconstructed from the spontaneous decay of an excited qubit. This requires a protocol in which: (i) the cavity is decoupled $\kappa \rightarrow 0$, (ii) the qubit is excited, (iii) all couplings are switched on for a brief period of time, and (iv) the excited population of the qubit is measured in a nondestructive way [40]. Alternatively, it is possible to relate the spectral function to the qubit spectroscopy by studying the low-power transmission spectrum of the cavity and relating the total line shape to the spectral function using the theory from Ref. [26].

\section{CONCLUDING REMARKS}

We have studied a cavity QED model beyond the standard perturbative treatment of losses using numerical and analytical techniques that apply for computing both the spectrum and the dynamics. Our paper builds on the polaron Hamiltonian [26] and matrix-product state simulations. In the former case, we have shown that techniques to solve the RWA and weak noise regime [as a Wigner-Weisskopf or $S(\omega)$ calculation] can 
be extended to work with a variety of regimes-USC, weak, strong coupling, etc.,- -

As concrete applications, we have discussed in detail the case of a two-level system that couples ultrastrongly to both the cavity and the bath - i.e., both $g$ and $\gamma$ are comparable to the qubit and cavity resonances. Using our techniques, we prove that strong dissipation renormalizes the qubit frequency, leading to a new resonance $\Omega=\Delta_{r}$ and changing its decay rate $\gamma_{r}$. Our simulations show that this renormalized decay rate can be used to define the onset of Rabi oscillations ( $g \cong$ $\left.\left|\gamma_{r}-\kappa\right| / 4\right)$ in a formula that extends beyond the RWA for all ranges of parameters. This suggests a regime where light and matter are ultrastrongly coupled, but losses are large enough to suppress Rabi oscillations. We call this regime the WUSC [cf. Fig. 1].

This paper has different possible continuations. On the experimental side, we have shown that all regimes and physics shown in this paper can be probed using state-of-the-art circuit QED technology. On the theoretical side, our numerical methods open the door to extend those experiments to study very challenging cavity QED phenomena, such as transmissionreflection experiments, Dicke physics, or nonlinear optics in the USC and WUSC regimes-enabling ultrasfast broadband photon sources, opening access to stronger nonlinearities and facilitating the study of non-Markovian open quantum systems among other phenomena to be explored. In these examples, the theoretical techniques may need to go beyond the first excited states, which lie in the single-excitation manifold in the polaron picture. Fortunately, the techniques used here (the MPS and the polaron Hamiltonian) already have shown their utility in working with higher excited states in spin-boson-like models [41-43].

\section{ACKNOWLEDGMENTS}

We would like to thank J. Aizpurua, A. Bermudez, and L. Martín-Moreno for inspiring discussions. We acknowledge support by the Spanish Ministerio de Ciencia, Innovación y Universidades within Project No. MAT2017-88358C3-1-R and No. FIS2015-70856-P. The Aragón Government project Q-MAD and CAM PRICYT Research Network QUITEMAD+ S2013/ICE-2801. EU-QUANTERA project SUMO is also acknowledged.

\section{APPENDIX A: BATH DISCRETIZATION}

In this appendix we explain how to discretize the bath for performing the numerical simulations.

\section{The Ohmic case}

In the spin-boson model,

$$
H=\frac{\Delta}{2} \sigma^{z}+\sigma^{x} \sum_{k}\left(g_{k} a_{k}^{\dagger}+\text { H.c. }\right)+\sum_{k} \omega_{k} a_{k}^{\dagger} a_{k},
$$

the bosonic bath is fully characterized by the spectral density, defined here as

$$
J(\omega):=2 \pi \sum_{k}\left|g_{k}\right|^{2} \delta\left(\omega-\omega_{k}\right) .
$$

In the Ohmic case $[J(\omega)=\pi \alpha \omega]$ the spontaneous emission rate in the Markovian regime is given by

$$
\Gamma=J(\Delta)=\pi \alpha \Delta .
$$

The dimensionless parameter $\alpha$ quantifies the spin-bosoncoupling strength.

To find a discretization, i.e., a finite set of coupling constants $g_{k}$, we first rewrite the sum in (A2),

$$
J(\omega)=2 \pi \sum_{k} \Delta \omega_{k} \frac{\left|g_{k}\right|^{2}}{\Delta \omega_{k}} \delta\left(\omega-\omega_{k}\right),
$$

so that

$$
J(\omega)=2 \pi \frac{\left|g_{k}\right|^{2}}{\Delta \omega_{k}},
$$

with $\Delta \omega_{k}$ as the frequency interval around $\omega_{k}$. When a frequency is degenerate $\omega_{k} \simeq \omega_{k^{\prime}}$, we have to add up all contributions coming from the different couplings.

A transmission line is a model for an Ohmic bath. Its discrete version is a set of coupled harmonic oscillators,

$$
H_{\mathrm{TL}}=\sum \frac{p_{i}^{2}}{2 \Delta x}+\sum \frac{\left(x_{i}-x_{i+1}\right)^{2}}{2 \Delta x} .
$$

The normal modes are known. In the chiral $k \geqslant 0$ case,

$$
k=\Delta k\{0,1, \ldots, N\}
$$

where the momentum spacing relates to $\Delta x$,

$$
\Delta k=\frac{2 \pi}{(2 N+1) \Delta x},
$$

which itself dictates the cutoff,

$$
\Delta x=\frac{2 v}{\omega_{c}} .
$$

The dispersion relation is then simply obtained from the speed of light $(v=1)$,

$$
\omega_{k}=\omega_{c} \sin (\Delta x k / 2) .
$$

In our model for the TLS $\Delta \omega_{k}=v \Delta k=\Delta k$ so that using (A5) and (A3),

$$
g_{k}=\sqrt{\frac{\alpha \Delta \Delta k}{2}} \sqrt{\omega_{k}}=\sqrt{\frac{\Gamma \Delta \Delta k}{2 \pi}} \sqrt{\omega_{k}},
$$

which are the couplings used in our numerical simulations. In Fig. 6 we compare the continuum spectral density and this discretization. The agreement is clear.

\section{The cavity-bath case}

The cavity-bath model is the positively defined quadratic Hamiltonian,

$$
H=\frac{1}{2} P^{2}+\frac{1}{2} \Omega^{2} X^{2}+\sum_{k} \frac{1}{2} p_{k}^{2}+\frac{1}{2} \omega_{k}^{2}\left(x_{k}-\frac{c_{k}}{\omega_{k}} X\right)^{2} .
$$

This is nothing but the Caldeira-Legget model of dissipation [22]. The spectral density of the cavity bath is given by

$$
J_{\text {cav }}=\frac{\pi}{2 \Omega} \sum \frac{c_{k}^{2}}{\omega_{k}} \delta\left(\omega-\omega_{k}\right) .
$$




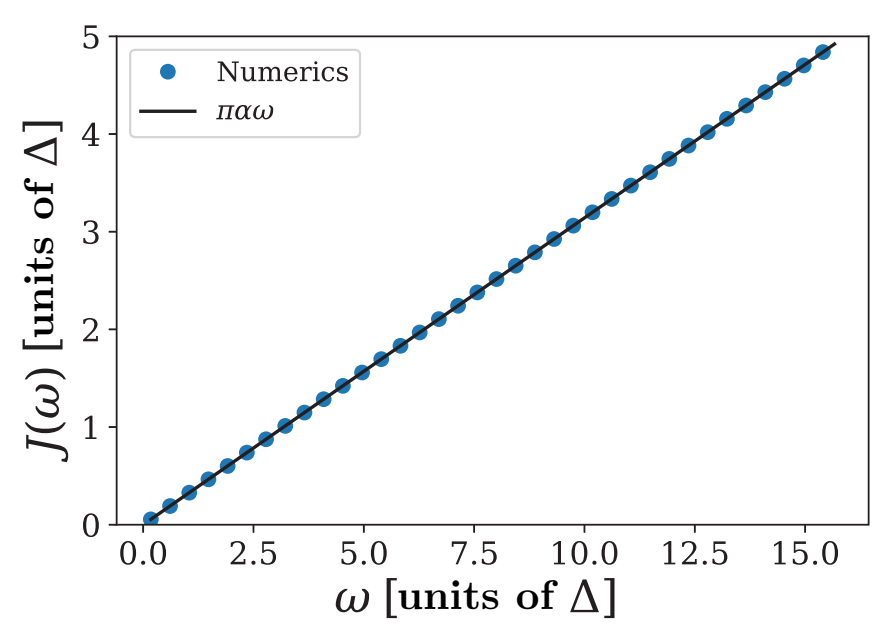

FIG. 6. Numerical discrete (circles) versus continuum Ohmic $[J(\omega)=\pi \alpha \omega]$ bath. The parameters used are $\alpha=0.1$ and $N=128$ bath modes. For aesthetic reasons, only every five points are plotted.

This expression ensures that $\Gamma_{\text {cav }}=J(\Omega)$ [cf. Eq. (A3)]. The differences between this last expression and (A2) arise because (A12) is written in terms of positionlike operators and in the spin boson in terms of annihilation-creation operators.

The Caldeira-Legget model can be rewritten as

$$
H=\frac{1}{2}\left(\mathbf{P}^{T} \mathbf{P}+\mathbf{X}^{T} B \mathbf{X}\right)
$$

with the matrix,

$$
B=\left(\begin{array}{cccc}
\Omega^{2}+\sum_{k} \frac{c_{k}^{2}}{\omega_{k}^{2}} & -c_{1} & \cdots & -c_{N} \\
-c_{1} & \omega_{1}^{2} & 0 & 0 \\
\cdots & 0 & \cdots & 0 \\
-c_{N} & 0 & 0 & \omega_{N}^{2}
\end{array}\right) .
$$

This model can be further diagonalized using a unitary transformation $U$ and eigenvalues $\Omega^{2}$ such that

$$
B=U \hat{\omega}^{2} U^{T}
$$

to give

$$
H=\frac{1}{2} \hat{\mathbf{P}}^{T} \hat{\mathbf{P}}+\frac{1}{2} \hat{\mathbf{X}} \hat{\omega}^{2} \hat{\mathbf{X}}
$$

We quantized the model as usual,

$$
\hat{X}_{j}=\sqrt{\frac{1}{2 \hat{\omega}_{j}}}\left(c_{i}+c_{i}^{\dagger}\right)
$$

obtaining an expansion for the original cavity mode,

$$
X=(U \hat{\mathbf{X}})_{0}=\sqrt{\frac{1}{2}} \sum_{j=1}^{N+1}\left(U \hat{\omega}^{-1 / 2}\right)_{1 j}\left(c_{i}+c_{i}^{\dagger}\right)
$$

In doing so, the qubit-cavity coupling can be written as the spin-boson coupling,

$$
\begin{aligned}
g \sigma^{x}\left(a+a^{\dagger}\right) & =g \sqrt{2 \Omega} \sigma^{x} X \\
& =g \sigma^{x} \sum_{j=1}^{N+1}\left(U \sqrt{\frac{\Omega}{\hat{\omega}}}\right)_{1 j}\left(c_{i}+c_{i}^{\dagger}\right) .
\end{aligned}
$$

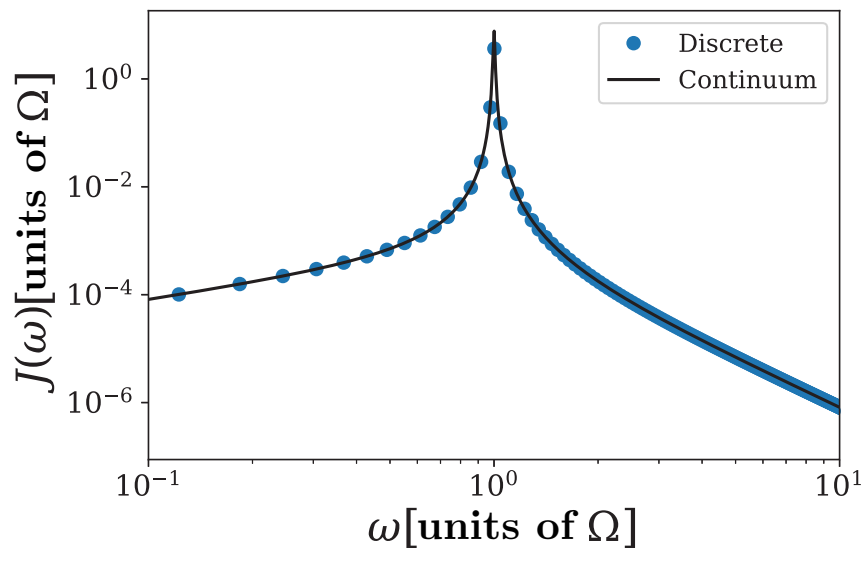

FIG. 7. Numerical discrete (circles) versus peaked spectral density continuum bath. The parameters used are $g=0.2, \kappa=0.01$, and $N=128$ modes.

The bath frequencies are given by the eigenvalues $\hat{\omega}_{i}$. As we have performed with the Ohmic spectral density, in Fig. 7 we compare the discrete model with the continuum one. The expression for the continuum case is explained in the next appendix [see Eq. (B17)]. Again, the agreement is clear.

\section{APPENDIX B: EXACT DIAGONALIZATION AND EFFECTIVE SPECTRAL DENSITY}

We have explained how to diagonalize the cavity and its bath numerically. It turns out that this task can be also performed analytically [44-47]. As a result, the diagonalized cavity mode plus bath can be characterized by a effective spectral density [25]. We summarize here, in a unified way, both the diagonalization and the effective spectral density.

\section{Exact diagonalization}

The eigenvalue problem (A14) reads

$$
\begin{aligned}
\Omega^{2} X-\sum c_{k} x_{k}+\sum_{k} \frac{c_{k}^{2}}{\omega_{k}^{2}} X & =\hat{\omega}_{j}^{2} X \\
\omega_{k}^{2} x_{k}-c_{k} X & =\hat{\omega}_{j}^{2} x_{k}
\end{aligned}
$$

Here, $\hat{\omega}_{j}^{2}$ are the eigenvalues [cf. Eq. (A17)]. From (B1b),

$$
x_{k}=\frac{c_{k}}{\hat{\omega}_{j}^{2}-\omega_{k}^{2}} X .
$$

Inserting the latter in (B1a) we obtain

$$
\Omega X+\left(\sum_{k} \frac{c_{k}^{2}}{\omega_{j}^{2}-\omega_{k}^{2}}+\frac{c_{k}^{2}}{\omega_{k}^{2}}\right) X=\hat{\omega}_{j}^{2} X .
$$

Therefore, the eigenvalues $\omega_{j}^{2}$ are the zeros of the function,

$$
g^{-1}(\omega)=\omega^{2}-\Omega^{2}-\left(\sum_{k} \frac{c_{k}^{2}}{\omega_{j}^{2}-\omega_{k}^{2}}+\frac{c_{k}^{2}}{\omega_{k}^{2}}\right) .
$$


Defining

$$
\mathcal{A}(\omega):=\left(\sum_{k} \frac{c_{k}^{2}}{\omega_{j}^{2}-\omega_{k}^{2}}+\frac{c_{k}^{2}}{\omega_{k}^{2}}\right)
$$

we rewrite $g^{-1}$ in a more convenient way, namely,

$$
g^{-1}(\omega)=\omega^{2}-\Omega^{2}-\mathcal{A}(\omega) .
$$

The zeros of $g^{-1}$ are the poles of $g$. It is important to note that the residues of $g$ are as follows:

$$
\operatorname{Res}\left(f, \omega_{j}\right)=\frac{1}{\left.\frac{\partial g^{-1}}{\partial \omega}\right|_{\omega=\omega_{j}}} .
$$

The orthogonal transformation (A16) fulfills the normalization condition,

$$
1=U_{0 j}^{2}+\sum_{k} U_{k j}^{2}=\left(1+\sum_{k} \frac{c_{k}^{2}}{\left(\omega_{j}^{2}-\omega_{k}^{2}\right)^{2}}\right) U_{0 j}^{2} .
$$

In the second equality, we have used (B2). Now, we note that [cf. Eq. (B4)]

$$
\frac{\partial g^{-1}(\omega)}{\partial \omega}=2 \omega\left(1+\sum_{k} \frac{C_{k}^{2}}{\left(\omega^{2}-\omega_{k}^{2}\right)^{2}}\right) .
$$

Using Eqs. (B7)-(B9), we arrive at

$$
U_{0 j}^{2}=\frac{2 \hat{\omega}_{j}}{\left.\frac{\partial g^{-1}}{\partial \omega}\right|_{\omega=\hat{\omega}_{j}}}=2 \hat{\omega}_{j} \operatorname{Res}\left(g, \hat{\omega}_{j}\right) .
$$

\section{Effective spectral density}

We can rewrite the spin-boson-coupling (A19) using (B10),

$$
g\left(U \sqrt{\frac{\Omega}{\hat{\omega}}}\right)_{1 j}=g \sqrt{\Omega} \sqrt{2 \operatorname{Res}\left(g, \omega_{j}\right)} .
$$

Therefore, the effective spectral density for the spin boson reads [cf. Eq. (A2)]

$$
J_{\text {eff }}(\omega)=2 \pi g^{2} \Omega \sum_{j} 2 \operatorname{Res}\left(g, \omega_{j}\right) \delta\left(\omega-\hat{\omega}_{j}\right) .
$$

Using now (B11) for any well-behaved function $f(\omega)$, we have that

$$
\begin{aligned}
\int J_{\mathrm{eff}}(\omega) f(\omega) & =4 \pi g^{2} \Omega \sum_{j} \operatorname{Res}\left(g, \omega_{j}\right) f\left(\omega_{j}\right) \\
& =4 \pi g^{2} \Omega \operatorname{Im}\left[\frac{1}{\pi} \int_{0}^{\omega_{c}} d \omega g\left(\omega-i 0^{+}\right)\right]
\end{aligned}
$$

Therefore,

$$
J_{\text {eff }}(\omega)=4 g^{2} \Omega \operatorname{Im}\left[g\left(\omega-i 0^{+}\right)\right] .
$$

So far, everything was general (we have not specified the spectral density for the cavity bath). We particularize to an Ohmic spectral density, see (A13) and (3b),

$$
J_{\text {cav }}(\omega)=\pi \alpha_{\text {cav }} \omega \rightarrow \kappa=J_{\text {cav }}(\Omega) .
$$

Now, we can compute $\mathcal{A}$ defined in (B5),

$$
\begin{aligned}
\mathcal{A}(\omega) & =\frac{2}{\pi} \Omega \int d \nu \frac{J(v) v}{\omega^{2}-v^{2}}-\frac{J(v)}{v} \\
& =\frac{2}{\pi} \Omega \alpha_{\mathrm{cav}} \int d \nu \frac{\omega^{2}}{\omega^{2}-v^{2}} \\
& =i \pi \Omega \alpha_{\mathrm{cav}} \omega=i \kappa \omega .
\end{aligned}
$$

Inserting the last result in the definition of $g^{-1}$, taking the imaginary part, and using (B14) we get

$$
J_{\text {eff }}(\omega)=\frac{4 g^{2} \kappa \omega}{\left(\Omega^{2}-\omega^{2}\right)^{2}+(\kappa \omega)^{2}},
$$

which is nothing but the peaked spectral density discussed in the main text and has been used to test our bath discretization [cf. Fig. (7)].

\section{APPENDIX C: SINGLE-EXCITATION TIME EVOLUTION (ANALYTICAL CALCULATIONS)}

We approximate Eqs. (12a) and (12b) as follows. We remove the term that is $O\left(f^{2}\right)$ and modify the rest, replacing the effective displacements $f_{k}$ with the original couplings $f_{k} \rightarrow c_{k}$. Besides, working in the rotated bases $\widetilde{\psi}=e^{i \Delta_{r} t} \psi$ and $\widetilde{\psi}_{k}=e^{i \Delta_{r} t} \psi_{k}$, these dynamical equations yield

$$
\begin{aligned}
\tilde{\psi} & =-i 2 \Delta_{r} \sum \widetilde{\psi}_{k} f_{k}, \\
\tilde{\psi}_{k} & =-i\left(\omega_{k}-\Delta_{r}\right) \widetilde{\psi}_{k}-i 2 \Delta_{r} c_{k} \widetilde{\psi},
\end{aligned}
$$

The set of amplitudes $\widetilde{\psi}_{k}$ can be formally integrated, and we replace the solutions in the equation for $\widetilde{\psi}$ arriving at the nonlocal differential equation,

$$
\dot{\widetilde{\psi}}=-\frac{1}{2 \pi} \int_{0}^{\infty} d \omega \int_{0}^{t} d \tau J(\omega) e^{i\left(\Delta_{r}-\omega\right)(t-\tau)} \widetilde{\psi}(\tau) .
$$

We recall that $J(\omega)$ is the sum of two contributions, one coming from the intrinsic TLS noise and the second coming from the cavity [cf. Eqs. (5)]. In the Markovian limit (which is consistent with the regime we are discussing), the Ohmic intrinsic TLS dissipation produces a local term,

$$
-\frac{1}{2 \pi} \int_{0}^{\infty} d \omega \int_{0}^{t} d \pi \psi \omega e^{i\left(\Delta_{r}-\omega\right)(t-\tau)} \widetilde{\psi}(\tau) \cong-\frac{\pi}{2} \alpha \Delta_{r} \widetilde{\psi} .
$$

The second summand is approximated with a Lorentzian. Using that $\kappa=\pi \alpha_{\text {cav }} \Omega$ [cf. Eqs. (5) and (3b)],

$$
\frac{4 g^{2} \kappa \Omega \omega}{\left(\Omega^{2}-\omega^{2}\right)^{2}+(\kappa \omega)^{2}} \cong \frac{g^{2} \kappa \Omega}{(\Omega-\omega)^{2}+\kappa^{2} / 4} .
$$

Because it is peaked around $\Omega$, we can extend the frequency integral $\int_{0}^{\infty} d \omega \rightarrow \int_{-\infty}^{\infty} d \omega$. We use the Fourier transform of the Lorentzian and back to the nonrotated picture ending up with

$$
\dot{\psi}=-g^{2} \int d t e^{i\left(\Delta_{r}-\Omega\right)(t-\tau)} e^{-\kappa|t-\tau| / 2} \psi(\tau)-\gamma \psi / 2 .
$$

Taking the time derivative, we have the local second-order differential equation,

$$
\ddot{\psi}=-\left(g^{2}-\gamma \kappa / 4-i \delta \gamma / 2\right) \psi-(\gamma+\kappa+2 i \delta) / 2 \dot{\psi},
$$


with $\delta=\Delta_{r}-\Omega$. The solution is $[\psi(0)=1$ and $\dot{\psi}(0)=$ $-\gamma / 2]$

$\psi=\frac{e^{-(1 / 4) t(\kappa+\gamma+\eta)}\left[(\gamma-\kappa)\left(1-e^{(1 / 2) \eta t}\right)+\eta+\eta e^{(1 / 2) t \eta}\right]}{2 \eta}$,

where

$$
\eta:=\sqrt{(\gamma-\kappa)^{2}-16 g^{2}}
$$

\section{APPENDIX D: QUBIT EMISSION SPECTRUM}

In computing the noise spectrum $S(\omega)$, several manipulations can be performed. It is convenient to solve (12a) and (12b) using the Laplace transform. We do it in the rotated frame $\widetilde{\psi}=e^{i \Delta_{r} t} \psi$ and $\widetilde{\psi}_{k}=e^{i \Delta_{r} t} \psi_{k}$. In the $s$ domain, the dynamical equations read

$$
\begin{aligned}
s \tilde{\psi}(s)-\widetilde{\psi}(0)= & -i 2 \Delta_{r} \sum_{k} \psi_{k}(s) f_{k}, \\
s \widetilde{\psi}_{k}(s)-\widetilde{\psi}_{k}(0)= & -i\left(\omega_{k}-\Delta_{r}\right) \widetilde{\psi}_{k}(s) \\
& -i 2 \Delta_{r} f_{k}\left(\widetilde{\psi}(s)+\sum_{k^{\prime}} f_{k^{\prime}} \widetilde{\psi}_{k^{\prime}}(s)\right) .
\end{aligned}
$$

Using that the qubit is initially excited, $\psi(0)=1$, and the bath is its ground-state $\psi_{k}(0)=0$, we find

$$
\widetilde{\psi}(s)=\frac{1}{s+\left(2 \Delta_{r}\right)^{2} \frac{\sum_{k} f_{k}^{2} /\left[s+i\left(\omega_{k}-\Delta_{r}\right)\right]}{1+i 2 \Delta_{r} \sum_{k} f_{k}^{2} /\left[s+i\left(\omega_{k}-\Delta_{r}\right)\right]}}
$$

which can be written as

$$
\widetilde{\psi}(s)=\frac{1}{s+K(s)}
$$

where

$$
K(s)=\left(2 \Delta_{r}\right)^{2} \frac{\mathcal{K}(s)}{1-i \mathcal{K}(s) / 2 \Delta_{r}},
$$

and

$$
\mathcal{K}(s)=\sum \frac{\left(2 \Delta_{r}\right)^{2} f_{k}^{2}}{s+i\left(\omega_{k}-\Delta_{r}\right)} .
$$

The $s$ domain is especially useful for computing the emission spectrum, that, in our case, is given by

$$
S(\omega)=\int_{0}^{\infty} d t \int_{0}^{\infty} d t^{\prime} e^{-i \omega\left(t-t^{\prime}\right)}\left\langle\sigma^{+}(t) \sigma^{-}\left(t^{\prime}\right)\right\rangle .
$$

In the single-excitation subspace, we have that

$$
\left\langle\sigma^{+}(t+\tau) \sigma^{-}(t)\right\rangle=\psi^{*}(t+\tau) \psi(t) .
$$

Using the inversion formula,

$$
f(t)=\frac{1}{2 \pi} \int_{-\infty}^{\infty} d \omega e^{i \omega t} f\left(i \omega+0^{+}\right),
$$

we get [cf. Eq. (D3)]

$$
\widetilde{\psi}(t)=\frac{1}{2 \pi} \int_{-\infty}^{\infty} d \omega \frac{e^{i \omega t}}{i \omega+0^{+}+K\left(i \omega+0^{+}\right)} .
$$

Since $\widetilde{\psi}=e^{i \Delta t} \psi$, the relevant object is as follows:

$$
\psi(t)=\frac{1}{2 \pi i} \int_{-\infty}^{\infty} d \omega \frac{e^{-i \omega t}}{\Delta_{r}-\omega-i K\left(i\left(\Delta_{r}-\omega\right)+0^{+}\right)}
$$

If we split in real and imaginary parts the kernel,

$$
K\left(i\left(\Delta_{r}-\omega\right)+0^{+}\right)=i[R(\omega)-i \Gamma(\omega)],
$$

together with (D7) and the definition (19), we realize that

$$
S(\omega) \sim \frac{1}{\left[\omega-\Delta_{r}-R(\omega)\right]^{2}+\Gamma(\omega)} .
$$

Thus, we just give explicit results for $G(\omega)$ and $\Gamma(\omega)$.

We use Eqs. (D4) and (D5),

$$
\begin{aligned}
\mathcal{K}\left(i\left(\Delta_{r}-\omega\right)+0^{+}\right) & =-i\left(2 \Delta_{r}\right)^{2} \int_{0}^{\infty} \frac{J(v)}{\left(v+\Delta_{r}\right)^{2}} \frac{1}{(v-\omega)-i 0^{+}} d v=\pi \frac{\left(2 \Delta_{r}\right)^{2} J(\omega)}{\left(\omega+\Delta_{r}\right)^{2}}-i\left(2 \Delta_{r}\right)^{2} \mathcal{P} \int_{0}^{\infty} \frac{J(v)}{\left(v+\Delta_{r}\right)^{2}} \frac{1}{(v-\omega)} d v \\
& \equiv \mathcal{K}^{\prime}-i \mathcal{K}^{\prime \prime} .
\end{aligned}
$$

With Eqs. (D4) and (D13), we have that [cf. Eq. (D11)]

$$
\begin{gathered}
R(\omega)=\frac{2 \Delta_{r}\left(\left(\mathcal{K}^{\prime}\right)^{2}+\left(\mathcal{K}^{\prime \prime}\right)^{2}-2 \Delta_{r} \mathcal{K}^{\prime \prime}\right)}{\left.\left(2 \Delta_{r}\right)^{2}-\mathcal{K}^{\prime \prime}\right)^{2}+\left(\mathcal{K}^{\prime}\right)^{2}}, \\
\Gamma(\omega)=\frac{\left(2 \Delta_{r}\right)^{2} \mathcal{K}^{\prime}}{\left(\left(2 \Delta_{r}\right)^{2}-\mathcal{K}^{\prime \prime}\right)^{2}+\left(\mathcal{K}^{\prime}\right)^{2}}
\end{gathered}
$$

\section{a. Calculations in the good cavity limit}

To produce analytical results we must solve the principal part in (D13). In the limit of good cavity $\alpha_{\text {cav }} \ll 1$ the integral can be performed analytically. Note that $\Delta_{r}$ acts as an effective cutoff. The integral with the peaked part of $J(\omega)$ cannot be performed, in general. However, if $\kappa$ is small enough, $J_{\text {eff }}$ is approximated by a Lorentzian (C4). Besides, we can approximate 
$v+\Delta_{r} \rightarrow \Omega+\Delta_{r}$ in the denominator. Putting everything together we have a close formula for $\mathcal{K}$,

$$
\mathcal{K}\left(i\left(\Delta_{r}-\omega\right)+0^{+}\right)=i 4 \Delta_{r}^{2}\left[\frac{R_{2}^{(0)}(\omega)}{\left(\Omega+\Delta_{r}\right)^{2}}+\frac{\gamma\left[\Delta_{r}+\omega+\omega \ln \left(\Delta_{r} / \omega\right)\right]}{\left(\Delta_{r}+\omega\right)^{2}}-i\left(\frac{\Gamma_{2}^{(0)}(\omega)}{\left(\Omega+\Delta_{r}\right)^{2}}+\frac{\pi \gamma \omega}{\left(\Delta_{r}+\omega\right)^{2}}\right)\right],
$$

where

$$
\begin{aligned}
& R_{2}^{(0)}(\omega)=\frac{g^{2}(\omega-\Omega)}{(\omega-\Omega)^{2}+(\kappa / 2)^{2}}, \\
& \Gamma_{2}^{(0)}(\omega)=\frac{g^{2} \kappa / 2}{(\omega-\Omega)^{2}+(\kappa / 2)^{2}} .
\end{aligned}
$$

[1] I. I. Rabi, Phys. Rev. 49, 324 (1936).

[2] E. Jaynes and F. Cummings, Proc. IEEE 51, 89 (1963).

[3] A. Frisk Kockum, A. Miranowicz, S. De Liberato, S. Savasta, and F. Nori, arXiv:1807.11636.

[4] P. Forn-Díaz, L. Lamata, E. Rico, J. Kono, and E. Solano, arXiv:1804.09275.

[5] D. Braak, Phys. Rev. Lett. 107, 100401 (2011).

[6] H.-P. Breuer and F. Petruccione, The Theory of Open Quantum Systems (Oxford University Press, Oxford, 2007).

[7] A. Rivas and S. F. Huelga, Open Quantum Systems (Springer, Berlin, Heidelberg, 2012).

[8] S. Ashhab and F. Nori, Phys. Rev. A 81, 042311 (2010).

[9] F. Beaudoin, J. M. Gambetta, and A. Blais, Phys. Rev. A 84, 043832 (2011).

[10] U. Weiss, Quantum Dissipative Systems, 2nd ed. (World Scientific, Singapore, 2008).

[11] I. de Vega and D. Alonso, Rev. Mod. Phys. 89, 015001 (2017).

[12] K. Le Hur, L. Henriet, L. Herviou, K. Plekhanov, A. Petrescu, T. Goren, M. Schiro, C. Mora, and P. P. Orth, C. R. Phys. 19, 451 (2017).

[13] I. Buluta, S. Ashhab, and F. Nori, Rep. Prog. Phys. 74, 104401 (2011).

[14] T. Niemczyk, F. Deppe, H. Huebl, E. P. Menzel, F. Hocke, M. J. Schwarz, J. J. Garcia-Ripoll, D. Zueco, T. Hümmer, E. Solano, A. Marx, and R. Gross, Nat. Phys. 6, 772 (2010).

[15] P. Forn-Díaz, J. Lisenfeld, D. Marcos, J. J. García-Ripoll, E. Solano, C. J. P. M. Harmans, and J. E. Mooij, Phys. Rev. Lett. 105, 237001 (2010).

[16] F. Yoshihara, T. Fuse, S. Ashhab, K. Kakuyanagi, S. Saito, and K. Semba, Nat. Phys. 13, 44 (2016).

[17] S. Bera, A. Nazir, A. W. Chin, H. U. Baranger, and S. Florens, Phys. Rev. B 90, 075110 (2014).

[18] G. Díaz-Camacho, A. Bermudez, and J. J. García-Ripoll, Phys. Rev. A 93, 043843 (2016).

[19] A. J. Leggett, S. Chakravarty, A. T. Dorsey, M. P. A. Fisher, A. Garg, and W. Zwerger, Rev. Mod. Phys. 59, 1 (1987).

[20] B. Peropadre, D. Zueco, D. Porras, and J. J. García-Ripoll, Phys. Rev. Lett. 111, 243602 (2013).

[21] P. Forn-Díaz, J. J. García-Ripoll, B. Peropadre, J.-L. Orgiazzi, M. A. Yurtalan, R. Belyansky, C. M. Wilson, and A. Lupascu, Nat. Phys. 13, 39 (2017).

[22] A. Caldeira and A. Leggett, Ann. Phys. (N.Y.) 149, 374 (1983).

[23] P. Hänggi and G.-L. Ingold, Chaos 15, 026105 (2005).

[24] G.-L. Ingold, Coherent Evolution in Noisy Environments (Springer, Berlin, Heidelberg, 2002), pp. 1-53.
[25] A. Garg, J. N. Onuchic, and V. Ambegaokar, J. Chem. Phys. 83, 4491 (1985).

[26] T. Shi, Y. Chang, and J. J. García-Ripoll, Phys. Rev. Lett. 120, 153602 (2018).

[27] A. W. Chin, Á. Rivas, S. F. Huelga, and M. B. Plenio, J. Math. Phys. 51, 092109 (2010).

[28] J. J. García-Ripoll, New J. Phys. 8, 305 (2006).

[29] E. K. Irish, Phys. Rev. Lett. 99, 173601 (2007).

[30] A. Auffèves, D. Gerace, J.-M. Gérard, M. França Santos, L. C. Andreani, and J.-P. Poizat, Phys. Rev. B 81, 245419 (2010).

[31] C. W. Gardiner and M. J. Collett, Phys. Rev. A 31, 3761 (1985).

[32] A. Ridolfo, S. Savasta, and M. J. Hartmann, Phys. Rev. Lett. 110, 163601 (2013).

[33] R. Stassi, S. Savasta, L. Garziano, B. Spagnolo, and F. Nori, New J. Phys. 18, 123005 (2016).

[34] H. J. Carmichael, R. J. Brecha, M. G. Raizen, H. J. Kimble, and P. R. Rice, Phys. Rev. A 40, 5516 (1989).

[35] X. Cao, J. Q. You, H. Zheng, and F. Nori, New J. Phys. 13, 073002 (2011).

[36] M. Haeberlein, F. Deppe, A. Kurcz, J. Goetz, A. Baust, P. Eder, K. Fedorov, M. Fischer, E. P. Menzel, M. J. Schwarz et al., arXiv:1506.09114.

[37] B. Peropadre, P. Forn-Díaz, E. Solano, and J. J. García-Ripoll, Phys. Rev. Lett. 105, 023601 (2010).

[38] Y. Yin, Y. Chen, D. Sank, P. O’Malley, T. White, R. Barends, J. Kelly, E. Lucero, M. Mariantoni, A. Megrant et al., Phys. Rev. Lett. 110, 107001 (2013).

[39] M. Pierre, I.-M. Svensson, S. Raman Sathyamoorthy, G. Johansson, and P. Delsing, Appl. Phys. Lett. 104, 232604 (2014).

[40] M. D. Reed, L. DiCarlo, B. R. Johnson, L. Sun, D. I. Schuster, L. Frunzio, and R. J. Schoelkopf, Phys. Rev. Lett. 105, 173601 (2010).

[41] E. Sánchez-Burillo, D. Zueco, J. J. García-Ripoll, and L. Martín-Moreno, Phys. Rev. Lett. 113, 263604 (2014).

[42] E. Sánchez-Burillo, J. García-Ripoll, L. Martín-Moreno, and D. Zueco, Faraday Discuss. 178, 335 (2015).

[43] V. Paulisch, T. Shi, and J. J. García-Ripoll, arXiv:1810.08439.

[44] P. Ullersma, Physica 32, 27 (1966).

[45] G. W. Ford, J. T. Lewis, and R. F. OConnell, J. Stat. Phys. 53, 439 (1988).

[46] V. Ambegaokar, J. Stat. Phys. 125, 1183 (2006).

[47] V. Ambegaokar, Ann. Phys. (N.Y.) 16, 319 (2007). 